\title{
SETS OF MULTIPLICITY AND DIFFERENTIABLE
} FUNCTIONS. II

BY

\author{
ROBERT KAUFMAN(1)
}

\begin{abstract}
The stability of certain sets of multiplicity is studied with reference to special classes of differentiable functions. Kronecker sets are produced as examples of instability. The most difficult theorem uses probability theory and an estimation of Kolmogoroff's $\epsilon$-entropy in a certain space of functions.
\end{abstract}

This paper investigates differentiable transformations of two classes of sets encountered in harmonic analysis, Kronecker sets and $M_{0}$-sets [4, Chapitre VII]; [5, Chapitre V]. The first theorem completes one proved in [7], and the second and third are then technical variations. The fourth is of a different nature and leaves open some difficult questions. By $C_{+}^{1}$ we denote the set of functions in $C^{1}[0,1]$ with positive derivatives.

THEOREM 1. Let $K$ be a compact subset of $C_{+}^{1}$, in the $C^{1}$-metric. Then there is a closed set $E \subseteq[0,1]$, such that each set $f(E), f \in K$, is an $M_{0}$-set, but $E$ can be transformed onto a Kronecker set by a mapping of the form

$$
\varphi(x)=4 x+\sum_{1}^{\infty} k^{-2} n_{k}^{-1} \sin n_{k} x
$$

After the proof of Theorem 3 it will be clear that $\varphi$ could be chosen so that $\varphi^{\prime}$ is monotone, or $\varphi^{\prime}$ has bounded variation and admits an absolutely convergent Fourier expansion. But an observation by Katznelson will show that both properties cannot always be attained.

The Riemann-Lebesgue lemma plays a major role in the following disguised form. (Henceforth $e(x) \equiv e^{2 \pi i x}$.) To estimate integrals $\int_{0}^{1} e(u f(t)) d t$, where $f$ is in $K$, we effect a change of variable, so the integral becomes

Received by the editors August 27, 1973 and, in revised form, January 14, 1974.

AMS (MOS) subject classifications (1970). Primary 42A72, 26A16; Secondary 42A36, $41 \mathrm{~A} 45,46 \mathrm{E} 10$.

Key words and phrases. Kronecker set, $M_{0}$-set, $C^{1}$ function, Riemann-Lebesgue lemma.

(1) Alfred P. Sloan Fellow. 


$$
\int_{a}^{b} e(u s) g^{\prime}(s) d s, \text { with } a=f(0), b=f(1),
$$

and the functions $g^{\prime}$ are equicontinuous and uniformly bounded. Thus the integrals tend to 0 as $u$ increases, uniformly with respect to $f$. If the derivatives $f^{\prime}$ are allowed to vanish, the conclusion is still valid for intervals $[r, s] \subseteq[0,1]$ on which $f^{\prime} \geqslant \delta$ or $f^{\prime} \leqslant-\delta$. In place of equicontinuity we can specify bounded variation, since the variation of $g^{\prime}$ is comparable with that of $f^{\prime}$ when $\left|f^{\prime}\right|$ $\geqslant \delta>0$.

1. Unfortunately a direct construction of $E$ and $\varphi$ appears hopeless, so we adopt some of the machinery used in [9]. (In spite of the apparent complications, the main point of the construction is just the Riemann-Lebesgue lemma as set forth above.) Thus $\left(F_{k}\right)$ is a dense sequence in the real Banach space $C^{d}[0,1]$, while $\left(g_{k}\right)$ is chosen in $C^{1}(T)$, the periodic version of $C^{1}$, and $g_{k}$ has meanvalue 1 and support $\left[-k^{-1}, k^{-1}\right]$ modulo 1 .

The idea of the construction is to form a sequence of absolutely continuous measure $\mu_{k}$, beginning with Lebesgue measure, so that

$$
\left|\int e(u f(x)) \mu_{k+1}(d x)-\int e(u f(x)) \mu_{k}(d x)\right| \leqslant(k+1)^{-4}
$$

for all real $u$ and all $f$ in $K$. Then the $w^{*}$-limit of $\mu_{k}$ is carried by a set $E$, and plainly $f(E)$ is an $M_{0}$-set for all $f$ in $K$. To obtain the last property of $E$ we write $\varphi_{k}$ for the $k$ th partial sum of the series representing $\varphi$ and $\mu_{k+1}=g_{k+1}\left(y_{k+1} \varphi_{k+1}-F_{k+1}\right) \mu_{k}$. Under the condition $\left|\varphi_{k}-\varphi\right|<\mid k^{-1} y_{k}^{-1}$, the $w^{*}$-limit is carried by a set $E$ on which $e\left(y_{k} \varphi-F_{k}\right) \rightarrow 0$ uniformly: $\varphi(E)$ is a Kronecker set.

Now $g_{k+1}$ is in $C^{1}(T)$ and has an absolutely convergent Fourier expansion $g_{k+1}(t)=\Sigma a_{n} e(n t)$, with $a_{0}=1$ (and $a_{n} \geqslant 0$ if there were any advantage in this). Hence the difference to be treated is a sum

$$
\sum^{\prime} a_{n} \int e(u f(x)) e\left(n y \varphi_{k+1}-n F_{k+1}\right) \mu_{k}(d x)
$$

where we have written $y=n_{k+1}$. Moreover $\mu_{k}$ is actually a $C^{1}$ function on $[0,1]$. Now $0<c_{1} \leqslant f^{\prime} \leqslant c_{2}$ for each $f$ while $2<\varphi_{k+1}^{\prime}<6$. Suppose that $\varphi_{k+1}$ were chosen first, and then $3 c_{2}|u| \leqslant|n| y$. We could write $n y \varphi_{k+1}-$ $u f= \pm n y \psi$, with $\psi$ in a certain compact subset of $C_{+}^{1}$, independent of $y$. Similarly, if $c_{1}|u| \geqslant 7|n| y$, we write $n y \varphi_{k+1}-u f= \pm u \psi$. In either case, the Riemann-Lebesgue lemma would apply for large $y$, because $|n| y \geqslant y$.

For the intermediate range, say $c_{3}|u| \leqslant|n| y \leqslant c_{4}|u|$, we construct $\varphi_{k+1}$, that is we choose $n_{k+1}$, to avoid the crossing of the graphs of certain derivatives. More exactly, $n y \varphi_{k+1}^{\prime}-u f^{\prime}=n y \psi^{\prime}$, with $\psi^{\prime}=(k+1)^{-2} \cos n_{k+1} x+G$, 
where $G$ is confined to a certain compact set $\Gamma$, independent of $k$. For simplicity we drop the factor $(k+1)^{-2}$, and write $Y=n_{k+1}$. Suppose $0<\eta<$ 1 and $Y$ is so large that $G$ oscillates at most $\eta$ on each interval of length $\pi Y^{-1}$. Then, if $[a, b]$ is such an interval and $x, x^{\prime}$ are solutions of the inequality $|\cos (Y x)+G(x)|<\eta, a \leqslant x, x^{\prime} \leqslant b$, then $\left|\cos \left(Y x^{\prime}\right)-\cos (Y x)\right|<2 \eta$. Thus the inequality defines a subset of $[0,1]$ of Lebesgue measure $\ll \eta^{1 / 2}$, say, uniformly for all $G$ in $\Gamma$. Equally important, the subset so defined is covered by $4 Y$ open intervals whose total measure is small. Because sets of small Lebesgue measure have small $\mu_{k}$-measure, a proper choice of $Y=n_{k+1}$ enables us to obtain the necessary estimates. In order to obtain the inequality $\left|\varphi_{k}-\varphi\right| y_{k}<k^{-1}$ we have only to ensure $y_{k+1}>k y_{k}$.

THEOREM 2. Let $L$ be the subset of $C^{1}[0,1]$ defined by the inequalities $1 \leqslant f^{\prime} \leqslant 2$ and $\operatorname{Var}\left(f^{\prime}\right) \leqslant 1$. Then Theorem 1 is valid with $L$ in place of K.

In the place of equicontinuity, we use integration by parts. The functions $\cos (Y x)+G$ are now characterized by an upper bound on the variation of $G$; with the aid of this upper bound, say $V$, we can estimate that $G$ varies by at most $A Y^{-1}$ on all but $A Y V$ of the intervals $\left[q \pi Y^{-1},(q+1) \pi Y^{-1}\right]-$ thus allowing only $A V \pi$ in Lebesgue measure. In fact, analysis of this kind leads to a stronger theorem.

THEOREM 2a. Let $w(k)>0$ and $\lim k w(k)=+\infty$ as $k \rightarrow+\infty$. Then the function $\varphi$ of Theorem 2 can be chosen so that $\varphi^{\prime}(x)=4+\Sigma k^{-2} u\left(n_{k}\right) \cos \left(n_{k} x\right)$. Consequently, if $h$ is positive on $[0, \infty)$ and $\lim h(u) u^{-1}=+\infty$ at $u=0+, \varphi$ can be formed so that $\left|\varphi^{\prime}(x)-\varphi^{\prime}(y)\right| \leqslant h(|x-y|)$ for small distances $|x-y|$.

2. The next theorem implicitly concerns Hausdorff measures and to that extent follows Ivašev-Musatov [3], and more directly [8, pp. 521-522].

THEOREM 3. Let $K$ be a compact subset of $C_{+}^{1}$. Then there is a function $\varphi$ in $C_{+}^{1}$, such that $\varphi^{\prime}$ is increasing and absolutely continuous, and a set $E$, such that $\varphi(E)$ is a Kronecker set, while each $f(E), f \in K$, is $M_{0}$.

In the following lemma, $h$ is a positive function on $(0,1)$ with $\lim \inf h(u)$ $=0$ at $0+, g \in C^{1}[0,1]$, and $\eta>0$.

Lemma. There exists a $C^{1}$-function $g_{1}$, whose graph is composed of approximately triangular sections over disjoint intervals $\left(a_{i}, b_{i}\right)$, so that

$$
\sum h\left(b_{i}-a_{i}\right)<\eta \text { and }\left|\int e(u f(x))\left[g_{1}(x)-1\right] g(x) d x\right|<\eta
$$

for all real $u$ and all $f$ in $K$. 
Proof. The interval $[0,1]$ can be divided into adjacent intervals $J_{1}, \cdots$, $J_{s}$, so small that $\int_{J}|g|<1 / 4 \eta$. Suppose that $g_{1}$ has been defined on $J_{1}, \cdots, J_{l}$ and that $g_{1}$ and $g_{1}^{\prime}$ vanish at the endpoints of these intervals. Putting $F=$ $J_{1} \cup \cdots \cup J_{l}$ we have by the equicontinuity

$$
\left|\int_{F} e(u f(x))\left[g_{1}(x)-1\right] g(x) d x\right|<\eta / 8
$$

when $f \in K$ and $|u|>U$. Now we divide $J_{l+1}$ into the adjacent intervals $R_{1}, \cdots, R_{n}$ whose length depends on $U$ in a manner to be specified momentarily. We form $g_{1}$ on $J_{l+1}$ out of triangular sections over the intervals $R_{1}$, $\cdots, R_{n}$. These must have the property that

$$
\int_{R} g_{1}(x) g(x) d x=\int_{R} g(x) d x
$$

and

$$
\int_{R}\left|g_{1}(x) g(x)\right| d x<2 \int_{R}|g(x)| d x
$$

for each $R$. To accomplish this, and for convenience in applying the lemma we specify that on the base of each triangle $c<g(x)<2 c$ or $c<-g(x)<2 c$. The key observation is that the bases of the triangles can be made arbitrarily small, once $R_{1}, \cdots, R_{n}$ are determined from $U$ and $K$. By the equicontinuity of the set $K$ we see that when the intervals are small enough, the error allowed in passing to $g_{1}(x) g(x)$ is at most $\eta / 4$, when $|u|<U$ and at most $\eta / 2$ for all $u$. By this inductive procedure we ensure that the error never reaches $\eta$. Because $\lim \inf h=0$, we can choose the subintervals $R_{1}, \cdots, R_{n}$ in $J_{l+1}$ so they contribute at most $2^{-l-1} \eta$ to the sum $\Sigma h\left(b_{i}-a_{i}\right)$. (For details, see [8, pp. 521-522].)

3. With the aid of the lemma we can prove Theorem 3 by modifying the foregoing analysis. Now we approximate $\varphi$ by sums $\varphi_{k}$, each of class $C^{2}$ with $\varphi_{k}^{\prime \prime}>0$. Our aim is to set $\varphi_{k+1}=\varphi_{k}+\psi$, with $\psi^{\prime \prime} \geqslant 0$ and $\int \psi^{\prime \prime}=k^{-2}$, for example. To choose $\psi$ we require an estimate of the $\mu_{k}$-measure of the sets defined by inequalities $\left|G+\psi^{\prime}\right|<k^{-3}$; as before $G$ is restricted to a compact subset $\Gamma$ of $C[0,1]$. Therefore we apply the lemma to a function $h$ that majorizes a common modulus of continuity for $\Gamma$; the number $\eta$ is to be determined later, but the change in the exponential integrals can be made as small as we please. At this step $\mu_{k}$ is replaced by a measure $\lambda=f_{1} \cdot \mu_{k}$, and now $\psi^{\prime}$ is defined temporarily to be piecewise-linear, constant between intervals $\left(a_{i}, b_{i}\right)$, and $\psi^{\prime}$ must increase on $\left(a_{i}, b_{i}\right)$ by $A h\left(b_{i}-a_{i}\right)$-of course, $A \Sigma h\left(b_{i}-a_{i}\right)=k^{-2}$. On an interval $\left(a_{i}, b_{i}\right)$ each function $G$ oscillates at most $h\left(b_{i}-a_{i}\right)$ while $\psi^{\prime}$ increases at least $k^{-2} \eta^{-1} h\left(b_{i}-a_{i}\right)$. Consequently the inequality $\left|G(x)+\psi^{\prime}(x)\right|<$ 
$h\left(b_{i}-a_{i}\right)$ defines a subinterval of $\left(a_{i}, b_{i}\right)$ of length at most $2 k^{2} \eta\left(b_{i}-a_{i}\right)$. Now the inequality on $g, c< \pm g<2 c$, valid on $\left(a_{i}, b_{i}\right)$, and the triangular shape of $g_{1}$ allow us to conclude that the $\lambda$-measure of the interval is at most $8 k^{2} \eta$ times the $|\lambda|$-measure of $\left(a_{i}, b_{i}\right)$, so the total $\lambda$-measure of the exceptional intervals can be made small, for all $G$, and of course the number of intervals does not depend on $G$. Once $\eta$ and $\psi^{\prime}$ are determined, we have only to adjust $\psi^{\prime}$ near the endpoints $a_{i}$ and $b_{i}$ to be of class $C^{\mathbf{1}}$. From this point onward the path is clear.

4. It is convenient to write $F L^{1}$ for the algebra of Fourier transforms of $L^{1}(-\infty, \infty)$, with the usual norm, often named $A$ or $W$.

THEOREM 3'. Theorem 3 remains true if we require $\varphi^{\prime}$ to be of bounded variation and in $F L^{1}$, but becomes false if we require $\varphi^{\prime}$ to be monotone and in $F L^{1}$.

Proof. To obtain the correct variant of Theorem 3 we replace the graph of $\psi^{\prime}$ over $\left(a_{i}, b_{i}\right)$ by a function of the form $a_{1} T\left(a_{2} t+a_{3}\right)$, where $a_{1}=$ $A \cdot h\left(b_{i}-a_{i}\right)$ as before, $a_{2}=\left(b_{i}-a_{i}\right)^{-1}$ and $a_{3}=-a_{i}\left(b_{i}-a_{i}\right)^{-1} . T$ vanishes off $[0,1]$, and $T=x(1-x)$ on $[0,1]$. Now the norm of the term just described depends only on $A \cdot h\left(b_{i}-a_{i}\right)$ and not on the length of the interval, and the same is true of its variation. For the Lebesgue measure of the set $\left|G(x)+\psi^{\prime}(x)\right|<h\left(b_{i}-a_{i}\right)$ the factor $k^{2} \eta$ is replaced by $O\left(k^{2} \eta\right)^{1 / 2}$, but this is of little importance.

To verify the negative part of Theorem $3^{\prime}$, we define the set $K \subseteq C_{+}^{1}$ by the relations

$$
f(0)=0, \quad 1 \leqslant f^{\prime} \leqslant 2,\left|f^{\prime}(x)-f^{\prime}(y)\right| \leqslant \Lambda(|x-y|),
$$

with $\Lambda$ monotone and $\Lambda(u) \equiv 1 / \log u^{-1}, 0<u \leqslant e^{-1}$. We shall prove that if $\varphi \in C_{+}^{1}, \varphi^{\prime}$ is monotone and $\varphi^{\prime}$ is in $F L^{1}$, then-assuming $E$ has measure 0 -there is an element $f$ of $K$, such that $\varphi$ coincides with a function $a(s) f(t)$ $+b(s)$, with $f$ in $K$, on a neighborhood of each element $s$ of $E \cap(0,1)$. From the Riemannian theory of $M_{0}$-sets, it is clear that $\varphi(E)$ must be $M_{0}$. In this construction of $f$ we assume that $\varphi^{\prime}$ is monotone on $[0,1]$ but not necessarily so elsewhere. This increases considerably the difficulty of the construction.

By an observation of Katznelson [4, p. 22], $(0,1)$ can be covered by compact intervals $I_{r}$, on each of which $\left|\varphi^{\prime}(x)-\varphi^{\prime}(y)\right|<\eta \Lambda(|x-y|)$, where $\eta$ is arbitrarily small, and will be determined as a function of the extreme values of $\varphi^{\prime}$. Next we can find a doubly infinite sequence $\cdots<a_{-1}<a_{0}<a_{1}<\cdots$ so that the inequality on $\left|\varphi^{\prime}(x)-\varphi^{\prime}(y)\right|$ is valid on each interval $\left[a_{j}, a_{j+1}\right]$, and the limits of $\left(a_{j}\right)$ are 0 and 1 . Let $L$ be linear on each segment $\left[a_{j}, a_{j+1}\right]$ 
and $L\left(a_{j}\right) \varphi^{\prime}\left(a_{j}\right)=1$. The slope of $L$ on $\left[a_{j}, a_{j+1}\right]$ does not exceed $\eta \Lambda\left(a_{j+1}-a_{j}\right) /\left(a_{j+1}-a_{j}\right) \cdot \inf \varphi^{\prime}$. Now $E$ has measure 0 , so we can find an approximation $L_{1}$, such that $L_{1}\left(a_{j}\right) \varphi^{\prime}\left(a_{j}\right)=1, L_{1}$ is constant on a neighborhood of each point in $E \cap(0,1)$, and on $\left[a_{j}, a_{j+1}\right]$ we have $\max \left|L_{1}^{\prime}\right| \leqslant 2 L^{\prime}$. Moreover, $L_{1}$ is monotone on each segment $\left[a_{j}, a_{j+1}\right]$. Thus $L_{1}(x) \varphi^{\prime}(x)$ has an acceptable modulus of continuity on each interval $\left[a_{j}, a_{j+1}\right]$, and equals 1 at each $a_{j}$. Hence the modulus of continuity on $[0,1]$ can be reduced to $\Lambda$ by taking $\eta$ small, and the same is true for the extreme values of $L_{1}(x) \varphi^{\prime}(x)$. Now we have only to set $f^{\prime}=L_{1} \varphi^{\prime}$.

It is remarkable that the modulus $\Lambda$ cannot be improved. In fact, if $\Lambda_{1}$ is monotone, $\lim \inf \Lambda_{1}(x) / \Lambda(x)=0$, and $K$ is defined with $\Lambda_{1}$ in place $\Lambda$, then the function $\varphi$ of Theorem 3 can be constructed so that $\varphi^{\prime}$ is monotone and $\varphi^{\prime} \in F L^{1}$. The first ingredient in proving this is the lemma to Theorem 3, applied to $h(x)=\Lambda_{1}(x) / \Lambda(x)$-in view of $\lim \inf h(x)=0$. Next we require a substitute for the triangular function used above and define it for $0<\delta<1 / 2$ :

$$
\begin{gathered}
S(\delta, x)=0 \text { on }(-\infty, 0] \text { and }[2,+\infty), \\
S(\delta, \delta)=S(\delta, 1)=1,
\end{gathered}
$$

and $S$ is linear on the intervals between $0, \delta, 1,2$. Then $\|S\|_{1} \leqslant 2, \operatorname{Var}(S)$ $\leqslant 2, \operatorname{Var}\left(S^{\prime}\right) \leqslant 5 \delta^{-1}$. These bounds yield $\|\hat{S}\|_{1}=O\left(\log \delta^{-1}\right)$, and this is exactly what is needed to achieve the construction of $\varphi^{\prime}$.

5. THEOREM 4. There exists a set $E \subseteq[0,1]$ such that $f(E)$ is an $M_{0^{-}}$ set for every $f$ in $C_{+}^{1}$ with $f^{\prime}$ in $F L^{1}$, but $E$ can be transformed onto a Kronecker set by a function $\varphi$ in $C_{+}^{1}$ and with $\varphi^{\prime}$ in every class $\operatorname{Lip}^{\alpha}, 0<$ $\alpha<1 / 2$.

Although this theorem does not attain the precision of the others, it is stronger in naming a specific metric property of $E$ that ensures the existence of many functions $\varphi$; in fact this property is a $C_{+}^{1}$-invariant. As $E \subseteq[0,1]$, each function $f$ in the theorem coincides on $E$ with a series $\Sigma a_{n} e^{i n \bar{x}}$, with $p(f) \equiv \Sigma(1+|n|)\left|a_{n}\right|<\infty$.

Lemma. Let $S_{1}$ be the set $p(f) \leqslant 1$ and $0<r<1 / 4$. Then any subset of $S_{1}$, whose elements have uniform distances at least $r$, contains at most $\exp A r^{-2 / 3} \log r^{-1}$ elements.

Proof. To each $f$ in $S_{1}$ we associate three functions $f_{a}, f_{b}$, and $f_{c}$ in $S_{1}$, whose sum is $f$. The sum of the Fourier series over the indices $n$, with $|n| r>4$, is $f_{a}$; the terms in which $|n| r \leqslant 4$ and $(1+|n|)\left|a_{n}\right| \leqslant r^{2 / 3}$ give $f_{b}$; and of course $f_{c}=f-f_{a}-f_{b}$. Now $\left|f_{a}\right| \leqslant r / 4$ and $f_{a}$ can be neglected entirely. Next $\left|f_{b}\right| \leqslant B r^{2 / 3} \log r^{-1}$ and $\left\|f_{b}^{\prime}\right\|_{L^{2}} \leqslant B r^{1 / 3}$. Thus the functions $r^{-1 / 3} f_{b}$ are uniformly bounded in uniform and $\mathrm{Lip}^{1 / 2}$-norm, and have uniformly bounded variation. By a theorem of Clements [2, p. 423], at most 
$\exp A r^{-2 / 3} \log r^{-1}$ of the functions $r^{-1 / 3} f_{b}$ can have mutual distances $>r^{2 / 3} / 4$. Finally, the remainder $f_{c}$ contains at most $r^{-2 / 3}$ terms in its Fourier expansion, and these are chosen among $<9 r^{-1}$ possible values of $n$. Thus $f_{c}$ is contained in one of $\exp A r^{-2 / 3} \log r^{-1}$ linear subspaces, each of real dimension at most $2 r^{-2 / 3}$; also $\left|f_{c}\right| \leqslant 1$. An elementary estimation of volumes in Euclidean space of dimension $2 r^{-2 / 3}$ yields a further factor $\exp A r^{-2 / 3} \log r^{-1} \quad[10$, p. 163].

6. The construction will be carried out for a fixed $\alpha$ in $(0,1 / 2)$, but the refinement necessary for the stronger conclusion will be plain. Let $m_{s}=$ $[(1+\alpha) s !+s]$ and $n_{s}=(s+1)$ ! for $s \geqslant 4$, let $M$ be the union of the intervals $\left[m_{s}, n_{s}\right]$, and $F$ the set of all sums $\Sigma_{M} \epsilon_{m} 2^{-m}\left(\epsilon_{m}=0\right.$ or 1$)$. To each $s$ there corresponds a splitting of $F$ into subsets of diameters at most $2 \cdot 2^{-m_{s+1}}$ and mutual distances at least $2^{-n_{s}-1}$. Observe that $m_{s+1}-(1+\alpha) n_{s} \rightarrow+\infty$, whence $F$ admits a diffeomorphism of class $\operatorname{Lip}^{1+\alpha}$ onto a Kronecker set, and so does every set that is $C_{+}^{1}$-equivalent to $F[6]$. In fact $E$ will be such a set.

To define this diffeomorphism, let us first of all introduce the notations $d_{s}=2^{-n_{s}}, N_{s}=2^{n_{s}-m_{s}}$ and $F_{s}$ for the part of $F$ corresponding to the interval $m_{s} \leqslant m \leqslant n_{s}$. Thus $F_{s}$ is a progression of $N_{s}$ terms and difference $d_{s}$, say $\left\{q d_{s}, 0 \leqslant q<N_{s}\right\}$. Suppose that $\left|y_{q}\right| \leqslant s^{-1} d_{s}, 1 \leqslant q \leqslant N_{s}$. Then $F_{s}^{*}$ is the sequence whose first term is 0 and $q$ th term is $(q-1) d_{s}+y_{1}+\cdots$ $+y_{q-1}, 0<q \leqslant N_{s}$. In an obvious manner $E=\Sigma F_{s}^{*}$ is a $C^{1}$ image of $F=$ $\Sigma F_{s}$, and is a $C_{+}^{1}$ image if we omit the displacements $y_{q}$ for a few initial values of $s$. Let $\mu_{s}$ be the uniform probability measure on $F_{s}^{*}$ and $\mu$ the convolution $\Pi * \mu_{s}$, so that $\mu$ is carried by $E$ We have to consider integrals $I(f, u)=$

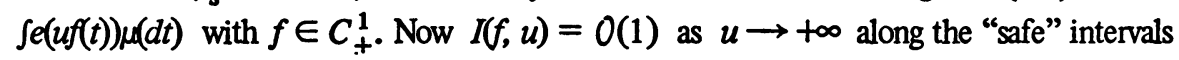
defined by the inequalities $u d_{s} \leqslant s^{-1}, u d_{s} N_{s} \geqslant s$; this is true for any $f$ in $C_{+}^{1}$ [7].

7. In dealing with the exceptional intervals $s^{-1} d_{s}^{-1} \leqslant u \leqslant(s+1) d_{s+1}^{-1} N_{s+1}^{-1}$, we encounter a technical difficulty: the condition $p(f) \leqslant 1$ implies nothing about the continuity properties of $f$ ! It is most convenient to dispose of this before entering upon detailed calculations. Suppose, then, that $\sigma$ is a probability measure on the hypercube $\Omega$ whose coordinates are the displacements $y_{q}$; to emphasize the dependence we write $I(f, \omega, u)$. The norm $p$ defines a separable Banach space, say $A^{1}$, and the functions $f$ belong to the open subset $A_{+}^{1}$ defined by the condition: $f^{\prime}>0$ on $[0,1]$. The set $Z$ on which $I(f, \omega, u) \neq O(1)$ is a Borel set in $A_{+}^{1} \times \Omega$. Following the method of criblage for analytic sets [1, pp. 121-149], we see that the projection of $Z$ into $\Omega$ can be approximated in $\sigma$-measure by projections of sompact subsets $K \subseteq Z$. Thus in proving that the projection of $Z$ has $\sigma$-measure 0 , we can assume that the functions $f$ in question have bounded norm $p(f)$-this much is trivial-and have equicontinuous derivatives on $[-\pi, \pi]$ with $f^{\prime} \geqslant \delta>0$ on an interval $[-\delta, 1+\delta]$. 
8. To define the numbers $y_{q}, 1 \leqslant q \leqslant N_{s}, s_{0} \leqslant s<\infty$, we take the random variable $X_{0}$ with density function $\sin ^{2} \pi x$ over $(-1,1)$ and 0 outside, and characteristic function $\Phi(y)$. Then the variables $y_{q}$ are mutually independent and $y_{q}$ has the distribution $s^{-1} d_{s} X_{0}$. It is familiar that the characteristic functions $\Phi\left(t N^{-1 / 2}\right)^{N}$ converge to that of a Gaussian variable, but it will be useful to ensure that the corresponding densities are smooth. Standard estimates of $\Phi$, near $\infty$ and near 0 , yield $\left|\Phi\left(t N^{-1 / 2}\right)\right|^{N}<A|t|^{-3}$ for real $t$ and $N=1,2,3, \cdots$. Therefore the densities generated by $\Phi\left(t N^{-1 / 2}\right)^{N}$ have uniformly bounded derivatives.

Next we use the inequality

$$
\left|\int e(u f(t)) \mu(d t)\right| \leqslant \sup \left|\int e(u f(t+v)) \mu_{s}(d t)\right|,
$$

where $0 \leqslant v \leqslant 1$. If $s$ is so large that $\mu_{s}$ carried by the interval $[0, \delta]$, then all the functions $t \rightarrow f(t+v)$ have equicontinuous derivatives $f^{\prime} \geqslant \delta$, over $0 \leqslant t \leqslant 1$. In estimating the integrals $\int e(u f(t)) \mu(d t)$, when $s^{-1} d_{s}^{-1} \leqslant u \leqslant$ $(s+1) d_{s+1}^{-1} N_{s+1}^{-1}$ we can admit an approximation $f^{*}$ to $f$ provided $\left|f^{*}-f\right|$ $<s^{-2} d_{s+1} N_{s+1}$, and we need only consider $s^{2} d_{s+1}^{-1} N_{s+1}^{-1}$ equally spaced numbers $u$. To estimate the number of functions $f^{*}$ that will be sufficient we use the lemma on approximation in $S_{1}$, the inequality $1+\alpha<3 / 2$, and the relations

$$
\log _{2} d_{s+1} N_{s+1} \cong-(1+\alpha)(s+1) !, \quad \log _{2} d_{s}=-(s+1) !
$$

The number of integrals to be checked separately is then $<\exp d_{s}^{-\beta}$ where $0<\beta<1$ (for large $s$ ). It must be emphasized that this number of integrals gives a good approximation to all the integrals $\int e(u f(t+v)) \mu_{s}(d t), 0 \leqslant v \leqslant 1$.

9. Finally we are ready to estimate the sums

$$
\sum e\left(u f\left(q d_{s}+y_{1}+\cdots+y_{q-1}\right)\right), \quad 0 \leqslant q<N_{s} .
$$

These we divide into $s^{6}$ partial sums, in each of which $q$ describes an arithmetic progression. Each of these sums can be abbreviated $\Sigma z_{r}, 0 \leqslant r<s^{-6} N_{s}$, and our aim is to show that all sums are $o\left(s^{-6} N_{s}\right)$ with probability very close to 1 .

We write $z_{r}^{*}$ for the conditional expectation of $z_{r}$, with respect to the $\sigma$-field of $z_{0}, \cdots, z_{r-1}$. A standard calculation yields

$$
E\left(\exp t\left|\sum z_{r}-z_{r}^{*}\right|\right) \leqslant 4 \exp A t^{2} s^{-6} N_{s}, \quad 0 \leqslant t \leqslant 1,
$$

whence $P\left(\left|\Sigma z_{r}-z_{r}^{*}\right|>\eta s^{-6} N_{s}\right)<4 \exp -\eta_{1} s^{-6} N_{s}$, where $\eta_{1}>0$ depends only on $\eta>0$. Now $s^{6} \exp -\eta_{1} s^{-6} N_{s} \exp d_{s}^{-\beta}$ decreases very rapidly as $s$ increases, because $\beta<1$ and $\log N_{s} \cong \log d_{s}^{-1}$. 
To end the proof we verify that $\left\|z_{r}^{*}\right\|_{\infty}=O(1)$ uniformly with respect to all the parameters; for this we need an analytic representation of $z_{r}^{*}$. Let $w^{1 / 2} \xi_{w}$ be the sum of $w=1,2,3, \cdots$ independent random variables with the law $X_{0}$. Then the conditional distribution of $z_{r}$, over the field of $z_{0}, \cdots$, $z_{r-1}$ can be realized by $e\left(u f\left(v+s^{-1} d_{s} s^{3} \xi_{s} 6\right)\right)$, where $v$ is measurable over the given field and $\xi_{s} 6$ is independent of $v$. But $\xi_{s} 6$ has a density function $g$ whose Fourier transform is $\Phi\left(t s^{-3}\right)^{s^{6}}$, so $z_{r}^{*}$ is given by $\int e\left(u f\left(v+s^{2} d_{s} t\right)\right) g(t) d t$. Now the integral of $g(t)$ over $|t|>M$ is at most $M^{-2}$ by Chebyshev's inequality. Over $[-M, M]$ we integrate by parts, using the inequality $u s^{2} d_{s} \geqslant d_{s}^{-1} \cdot s^{2} d_{s}=$ $s$ and the uniform boundedness of $g^{\prime}$. Moreover the compactness of the functions $f$ in $A_{+}^{1}$ implies the equicontinuity properties discussed before. To treat functions of the type $u f\left(a_{1}+a_{2} t\right)$, with $u a_{2}$ large and $a_{2}$ small we write them as $u a_{2} \cdot a_{2}^{-1} f\left(a_{1}+a_{2} t\right)$. Then Riemann-Lebesgue can be invoked to obtain a uniform limit.

\section{REFERENCES}

1. N. Bourbaki, Éléments de mathématique. I: Les structures fondamentales de l'analyse. Fasc. VIII. Livre III. Topologie générale. Chap. 9: Utilisation des nombres réels en topologie générale, Actualités Sci. Indust., no. 1045, Hermann, Paris, 1958. MR 30 \#3493.

2. G. F. Clements, Entropies of sets of functions of bounded variation, Canad. J. Math. 15 (1963), 422-432. MR 28 \#2190.

3. O. S. IvaSev-Musatov, $M$-sets and h-measures, Mat. Zametki 3 (1968), 441-447 = Math. Notes 3 (1968), 281-285. MR 37 \#3273.

4. J.-P. Kahane, Séries de Fourier absolument convergentes, Ergebnisse der Mathematik und ihrer Grenzgebiete, Band 50, Springer-Verlag, Berlin and New York, 1970. MR 43 \#801.

5. J.-P. Kahane and R. Salem, Ensembles parfaits et séries trigonométriques, Actualités Sci. Indust., no. 1301, Hermann, Paris, 1963. MR 28 \#3279.

6. R. Kaufman, $A$ functional method for linear sets, Israel J. Math. 5 (1967), 185187. MR $38 \# 4902$.

7. Sets of multiplicity and differentiable functions, Proc. Amer. Math. Soc. 32 (1972), 472-476.

8. Kronecker sets and metric properties of $M_{0}$-sets, Proc. Amer. Math. Soc. 36 (1972), 519-524. MR 46 \#9638.

9. - Topics on Kronecker sets, Ann. Inst. Fourier Grenoble (to appear).

10. G. G. Lorentz, Approximation of functions, Holt, Rinehart and Winston, New York, 1966. MR 35 \#4642.

DEPARTMENT OF MATHEMATICS, UNIVERSITY OF ILLINOIS, URBANA, ILLINOIS 61801 